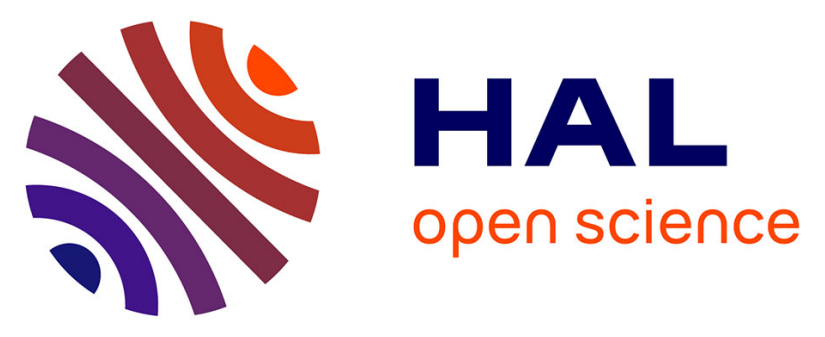

\title{
Estimation of Radiotherapy Dose Fields from a Few Projections: How Many Projections will Ensure Uniqueness?
}

\author{
Odran Pivot, Rolf Clackdoyle, Simon Rit, Laurent Desbat
}

\section{To cite this version:}

Odran Pivot, Rolf Clackdoyle, Simon Rit, Laurent Desbat. Estimation of Radiotherapy Dose Fields from a Few Projections: How Many Projections will Ensure Uniqueness?. 2020 IEEE Nuclear Science Symposium and Medical Imaging Conference (NSS/MIC), Oct 2020, Boston (virtual), United States. pp.1-4, 10.1109/NSS/MIC42677.2020.9507859 . hal-03344335

\author{
HAL Id: hal-03344335 \\ https://hal.science/hal-03344335
}

Submitted on 15 Sep 2021

HAL is a multi-disciplinary open access archive for the deposit and dissemination of scientific research documents, whether they are published or not. The documents may come from teaching and research institutions in France or abroad, or from public or private research centers.
L'archive ouverte pluridisciplinaire $\mathbf{H A L}$, est destinée au dépôt et à la diffusion de documents scientifiques de niveau recherche, publiés ou non, émanant des établissements d'enseignement et de recherche français ou étrangers, des laboratoires publics ou privés. 


\title{
Estimation of Radiotherapy Dose Fields from a Few Projections: How Many Projections will Ensure Uniqueness?
}

\author{
Odran Pivot, Rolf Clackdoyle, Simon Rit and Laurent Desbat
}

\begin{abstract}
The cross-section of the dose field generated by a linac with multi-leaf collimators (MLC) can be examined using scintillating fiber technology to obtain a few parallel projections of this (nearly) binary image. We examine ambiguity of the reconstructions when using only six projections, and demonstrate that unique solutions cannot be ensured. We suggest alternative approaches or conditions on the MLC configuration that would restore unicity.
\end{abstract}

\section{INTRODUCTION}

Multi-leaf collimators (MLCs) play an essential role in radiotherapy by shaping the treatment beam to the target tumour. The MLC consists of opposing pairs of rectangular 'leaves' whose role is to allow the beam to pass through a small rectangular region. The leaves can be slid along their long axis and fixed in the desired position for treatment. Typically, there are about 100 pairs of leaves. There are various methods of quality assurance, to verify that the delivered dose matches what was intended. A novel technique of measuring the dose field in real time is being developed in Lyon, using six layers of scintillating fibers with each layer providing a parallel projection of the beam cross-section at different angles [1]. Ignoring the effect of beam penumba and other (smaller) confounding effects, these 6 measurement functions are parallel projections of a constant dose function inside the shape defined by the collimator leaves. The objective is to determine (verify) the dose field, i.e., the dose intensity and the pattern of the collimator leaves. Here, we consider this binary image reconstruction problem, and ask whether the six projections provide enough information to uniquely determine the dose field. We provide illustrations of ambiguous projection data from realistic dose fields, and discuss options to avoid these scenarios.

\section{MATHEMATICAL DESCRIPTION}

For each of $N$ pairs of MLC leaves, we let $L_{i}, R_{i}$ (where $0 \leq L_{i} \leq R_{i}$ ) indicate the left and right endpoints respectively

Manuscript submitted December 19, 2020.

With financial support from ITMO Cancer AVIESAN (Alliance Nationale pour les Sciences de la Vie et de la Santé, National Alliance for Life Sciences $\&$ Health) within the framework of the Cancer Plan.

This work was performed within the framework of the LABEX PRIMES (ANR-11-LABX-0063) of Université de Lyon, within the program 'Investissements d'Avenir' (ANR-11-IDEX-0007) operated by the French National Research Agency (ANR).

O. Pivot, R. Clackdoyle, and L. Desbat are all with the Univ. Grenoble Alpes, CNRS, Grenoble INP, TIMC-IMAG, 38000 Grenoble, France.

S. Rit is with Univ. Lyon, INSA-Lyon, UCB Lyon 1, UJM Saint-Etienne, CNRS, Inserm, CREATIS, Centre Lyon Bérard, 69373 Lyon, France. of the $i$-th leaf-pair. By scaling the units if necessary, the distance between the centers of the leaves $i$ and $i+1$ is one. The binary image function $f$ is defined by

$$
f(x, y)= \begin{cases}d & y \in[i-1, i), x \in\left[L_{i}, R_{i}\right], i \in\{1, \ldots, N\} \\ 0 & \text { otherwise }\end{cases}
$$

where $d>0$ is the dose intensity. See Fig. 1 .
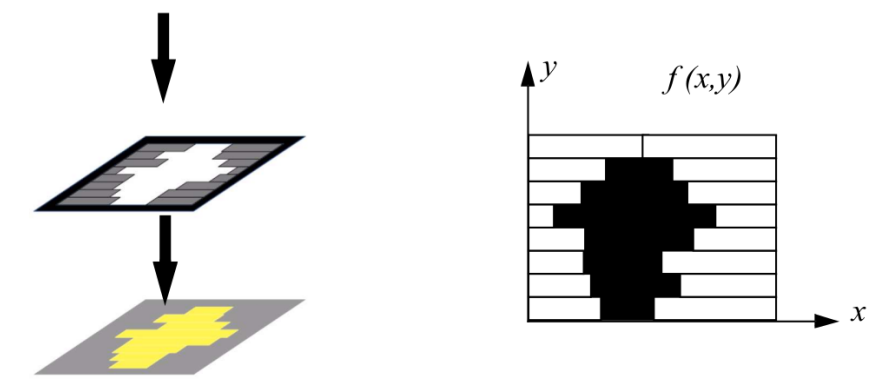

Fig. 1. Left: conceptual image of the therapy beam passing through the MLC and generating the yellow dose field. Right: the dose field is modelled by the binary function $f(x, y)$. Knowledge of $f(x, y)$ is equivalent to knowing the aperture positions $\left(L_{i}, R_{i}\right)$ of the rectangular collimators.

The projection of $f$ at angle $\phi$ is defined as usual by

$$
\mathcal{P}_{\phi} f(s)=\int_{-\infty}^{\infty} f\left(r \vec{u}_{\phi}+s \vec{v}_{\phi}\right) \mathrm{d} r
$$

where $\vec{u}_{\phi}=(\cos \phi, \sin \phi), \vec{v}_{\phi}=(-\sin \phi, \cos \phi)$ and $\phi \in$ $\Phi_{\alpha}=\left\{\phi_{1}+\alpha, \phi_{2}+\alpha, \ldots, \phi_{P}+\alpha\right\}$ where the angle $\alpha$ is unknown. Unless stated otherwise, the number of projections is $P=6$, with equally-spaced projection directions $\phi_{1}=$ $0^{\circ}, \phi_{2}=30^{\circ}, \ldots, \phi_{6}=150^{\circ}$.

The reconstruction problem is to estimate the $N$ leaf positions $\left(L_{i}, R_{i}\right)$, the dose $d$, and the offset angle $\alpha$ from the six measured projections $\phi \in \Phi_{\alpha}$. A general method was presented by Goulet et al [2]. A more restrictive one (for the case $N=1$ ) was presented by Desbat et al [3]. Here, we further simplify the problem by assuming that $d$ and $\alpha$ are known, so the problem is equivalent to just obtaining the binary image $f$ from the six projections. Our allowable binary images are $h$-convex (horizontally convex), which means that for each $y$, the support of the 1D function $f(\cdot, y)$ is convex. A similar definition applies for $v$-convex (vertically convex); observe that the example of Fig. 1 (right) is not v-convex. Finally an $h v$ convex binary image is one which is both $\mathrm{h}$ - and $\mathrm{v}$-convex. 

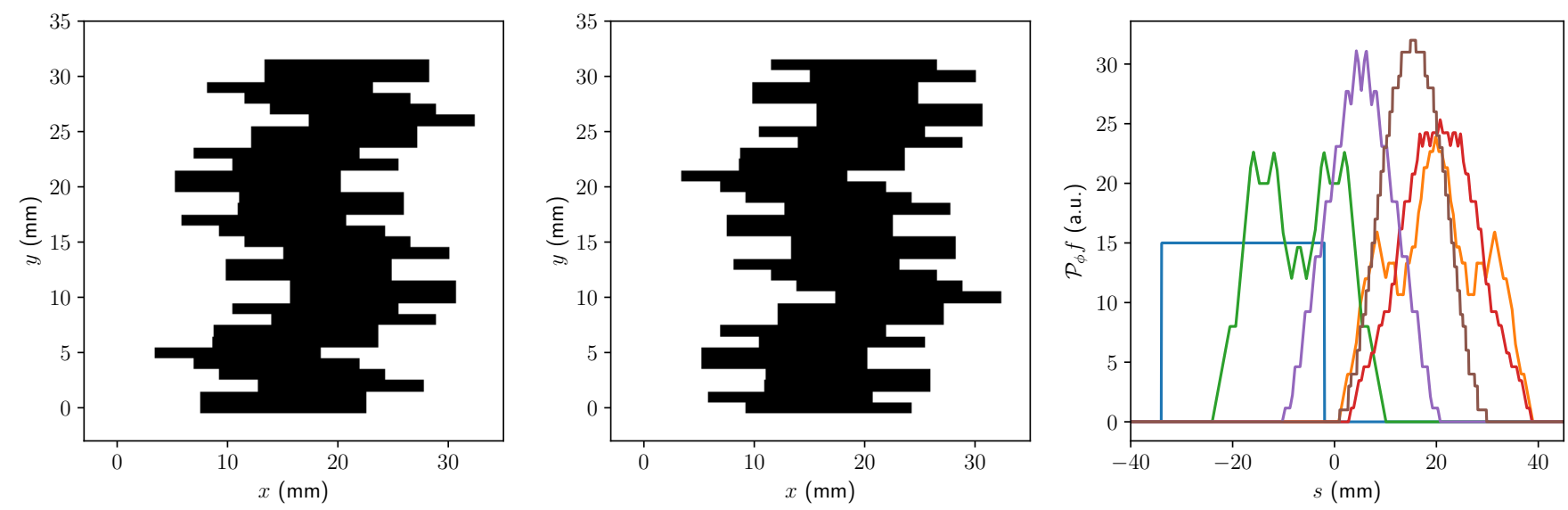

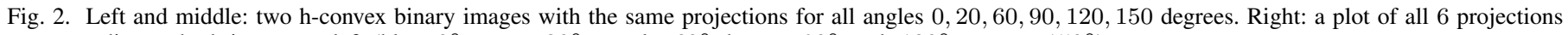
corresponding to both images at left (blue: $0^{\circ}$, green: $30^{\circ}$, purple: $60^{\circ}$, brown: $90^{\circ}$, red: $120^{\circ}$, orange: $150^{\circ}$ ).

\section{AN EXAMPLE}

Assume the known angle $\alpha$ is equal to zero. Fig. 2 shows two h-convex binary images whose six projections (for $\phi \in \Phi_{0}$ ) are exactly identical to each other. Therefore these two MLC configurations cannot be distinguished from their six projections.

It is conceivable that this non-uniqueness example arose because one of the projections is aligned with the horizontal direction (the direction of h-convexity), i.e. because $\alpha=0$. However, we describe the construction of this example below, to illustrate that similar situations can occur for any $\alpha$. Furthermore, similiar non-uniqueness examples can be constructed for any set of projection angles, and for any number of projections.

\section{GARDNER'S BUILDING RULE}

Given an arbitrary collection of projection angles $\Phi=$ $\left\{\phi_{1}, \phi_{2}, \ldots, \phi_{M}\right\}$, Gardner provides an algorithm to generate two binary images $C$ and $D$ with matched projections for all $\phi \in \Phi$, from two binary images $A$ and $B$ with matched projections for all $\phi \in \Phi \backslash\left\{\phi_{M}\right\}$ [4], [5]. The algorithm is illustrated in Fig. 3. We refer to it here as Gardner's Building Rule.

1) Let $A$ and $B$ be two compact subsets of the plane such that:

$$
\mathcal{P}_{i} A=\mathcal{P}_{i} B, \quad 1 \leq i \leq M-1 .
$$

(In this abuse of notation, $\mathcal{P}_{i} A$ means $\mathcal{P}_{\phi_{i}} f_{A}$ where $f_{A}$ is the indicator function of $A$, i.e. the corresponding binary image of $A$.)

2) Let $A^{\prime}$ and $B^{\prime}$ be the respective translations of $A$ and $B$ by any vector $\vec{w}$ :

$$
A^{\prime}=A+\vec{w} ; B^{\prime}=B+\vec{w} .
$$

Because the same translation, $\vec{w}$, was given to both $A$ and $B$, we still have:

$$
\mathcal{P}_{i} A^{\prime}=\mathcal{P}_{i} B^{\prime}, \quad 1 \leq i \leq M-1 .
$$

3) If we choose $\vec{w}=\lambda \vec{u}_{M}$ (for any nonzero $\lambda$ ), then:

$$
\mathcal{P}_{M} A^{\prime}=\mathcal{P}_{M} A ; \mathcal{P}_{M} B^{\prime}=\mathcal{P}_{M} B
$$

4) Finally, selecting $\lambda$ to be large enough to avoid overlaps, define $C=A \cup B^{\prime}$ and $D=A^{\prime} \cup B$. Then:

$$
\mathcal{P}_{i} C=\mathcal{P}_{i} D, \quad 1 \leq i \leq M
$$

(7) can easily be verified by first considering $1 \leq i \leq M-1$ and then checking for $i=M$, noting that for all $i, \mathcal{P}_{i} C=$ $\mathcal{P}_{i} A+\mathcal{P}_{i} B^{\prime}$, and similarly for $D$.

Note that Gardner's building rule can be directly generalized to any objects $A$ and $B$ (i.e. $f_{A}$ and $f_{B}$ could be any realvalued density functions).

\section{NON-UNIQUENESS OF H-CONVEX SETS WITH 6 PROJECTIONS}

Gardner's building algorithm is too general for our purposes, so we adapted it to ensure that we would obtain h-convex sets that were connected. These objectives were achieved using a carefully chosen initial configuration, and by ensuring the displacement vectors directed a vertical motion that added open leaves at the next available row. For the example of Fig. 2, the initial configuration for object $A_{1}$ was a rectangle of height 1 and width 15 centered at $x=15$, and $B_{1}$ was the same rectangle centered at $x=16.75$ so clearly $\mathcal{P}_{1} A_{1}=\mathcal{P}_{1} B_{1}$ since $\phi_{1}=0^{\circ}$ (horizontal projection). The second angle used was $\phi_{2}=150^{\circ}$, with the displacement chosen as $\lambda_{2} \vec{u}_{2}=\left(2 \cot 150^{\circ}, 2\right)$, and the rest of the sequence was $\lambda_{3} \vec{u}_{3}=\left(4 \cot 30^{\circ}, 4\right), \lambda_{4} \vec{u}_{4}=\left(8 \cot 120^{\circ}, 8\right), \lambda_{5} \vec{u}_{5}=$ $\left(16 \cot 60^{\circ}, 16\right), \lambda_{6} \vec{u}_{6}=(0,32)$. Fig. 4 shows the two objects $A_{n}$ and $B_{n}$ along with the $n$ projections at each of the six steps of the algorithm. Taking a wide enough starting starting image ensured that each step generated a single connected image. More h-convex binary images with the same projections as in Fig. 2 can be generated either by applying the angles in a different order, or by a new center for the first rectangle. We note also that the total height of the binary image is 32 .

More importantly, this same procedure can be applied for any $\Phi_{\alpha}$, i.e. for the same set of 6 projection angles with an offset by $\alpha$ (such as $10^{\circ}, 40^{\circ}, 70^{\circ}, 100^{\circ}, 130^{\circ}, 160^{\circ}$ ). In summary, for any six projection angles, it is easy to generate 


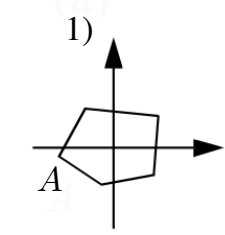

2)
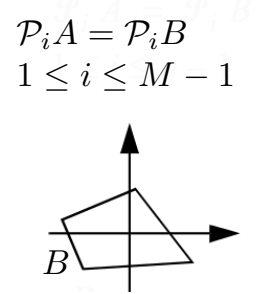

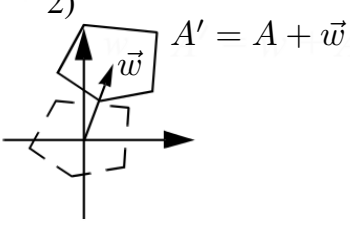

$\mathcal{P}_{i} A^{\prime}=\mathcal{P}_{i} B^{\prime}$

$1 \leq i \leq M-1$

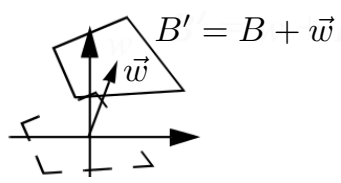

3)
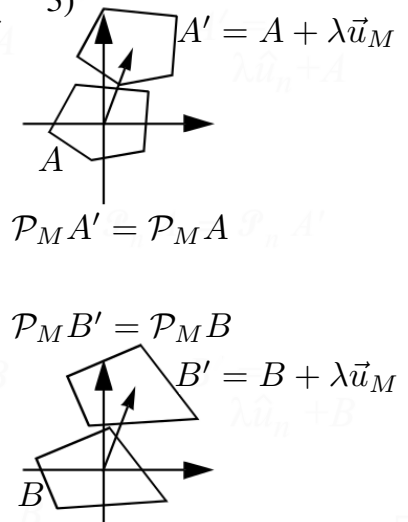
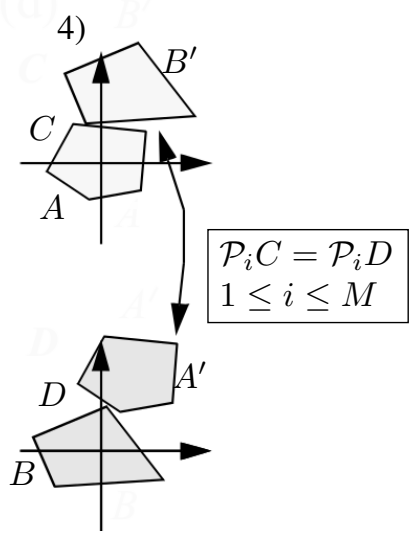

Fig. 3. Constructing binary objects $C$ and $D$, with $M$ matched projections, from $A$ and $B$ which have $M-1$ matched projections. Translate $A$ in the $\vec{u}_{M}$ direction to create $A^{\prime}$. Make the same translation of $B$ to create $B^{\prime}$. Finally, $C=A \cup B^{\prime}$ and $D=B \cup A^{\prime}$.
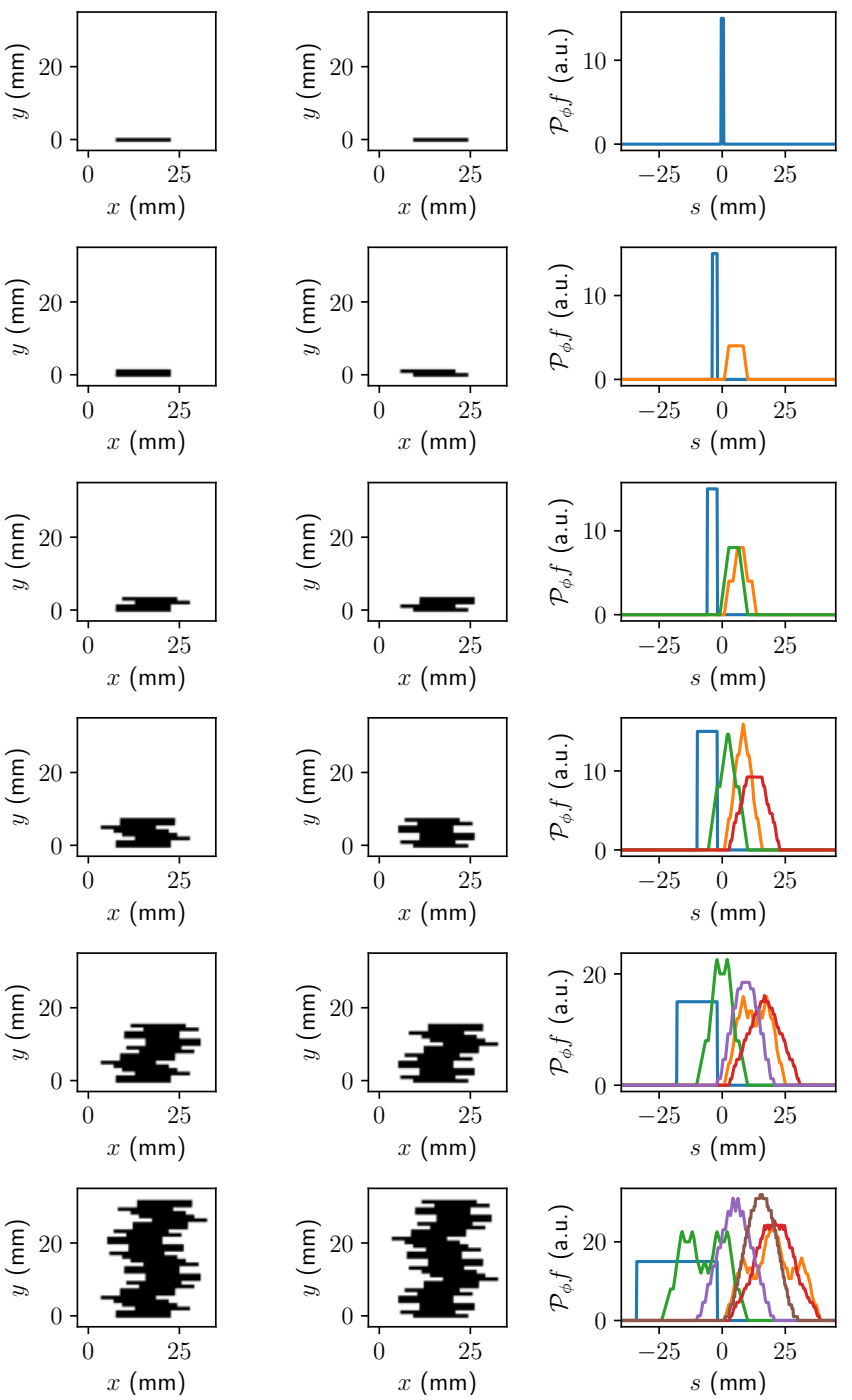

Fig. 4. Each of the six steps of the algorithm for generating the example of Fig. 2. Left and middle columns, $n$-th row: the two h-convex images $A_{n}$ and $B_{n}$ with the same $n$ projections shown at right (blue: $0^{\circ}$, green: $30^{\circ}$, purple: $60^{\circ}$, brown: $90^{\circ}$, red: $120^{\circ}$, orange: $150^{\circ}$ ). examples of non-uniqueness, even for just $N=32$ pairs of leaves.

\section{RESTRICTION TO CONVEX OR HV-CONVEX SETS}

We note that the example of Fig. 2 has very ragged sides, and perhaps normal MLC setting would be more smooth, or even approximate a convex shape. We ask if the six projections can uniquely define the dose field, assuming it belongs to some restricted set of h-convex images. A number of results can be found in Gardner's book [5]. Any seven distinct projection directions will uniquely determine a convex binary image, or a convex lattice, but there are examples (Fig. 5) of six directions for which uniqueness fails [6] (see also the website www.geometrictomography.com). This example, shown in Fig. 5 , is lattice-convex so it is also hv-convex. However, the construction does not appear to generalize to the six directions in $\Phi_{\alpha}$. Reducing consideration to only hv-convex sets is not likely to be restrictive enough. A slightly more restrictive class is the Q-convex sets, for which similar uniqueness results are known to hold [7].

As an alternative to considering more restrictive classes of dose fields, we recommend moving from $P=6$ to $P=8$ projections. In this way, we immediately achieve uniqueness for any lattice-convex set, and also Q-convex sets. The construction of Fig. 2 would still generate many non-unique examples but they would all require a minimum of $N=128$ pairs of leaves which may be more than the number on some linac machines. Less restrictive dose fields, such as hv-convex fields would have to be investigated for uniqueness from 8 projections.

\section{DISCUSSION AND CONCLUSIONS}

We have examined the uniqueness question for reconstruction of dose fields (h-convex sets) from 6 projections. We demonstrated substantial non-uniqueness, with an algorithm to generate multiple binary images with the same projections. Restricting the class of dose fields to only hv-convex sets, Q-convex sets or even lattice-convex sets does not appear to recover uniqueness; we do not currently have a definitive answer for such cases. In practice, we would recommend 

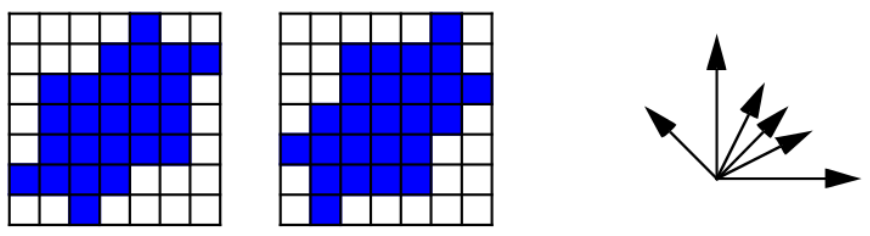

Fig. 5. Example of non-uniqueness of two hv-convex sets with the same six projections from angles $0^{\circ}, 45^{\circ}, 90^{\circ}, 26.6^{\circ}, 63.4^{\circ}$ (the last two angles are $\arctan 0.5$ and $\arctan 2$ ). The six directions are indicated at right with arrows.

using 8 projections for which various convexity constraints immediately impose uniqueness. The method of generating general non-unique h-convex sets still applies but requires at least 128 pairs of leaves. For hv-convex sets, unicity with 8 projections is an open question.

Including penumbra effects using convolutions by gaussian distributions does not change the non-uniqueness examples.

\section{REFERENCES}

[1] P. Pittet, J. Esteves, J.-M. Galvan, G.-N. Lu, F. Blanc, G. Haefeli, P. Hopchev, S. Rit, L. Desbat, J. Ribouton, and P. Jalade, "SciFi detector and associated method for real-time determination of profile and output factor for small fields in stereotactic radiotherapy," Medical Physics, vol. 47, no. 4, pp. 1930-1939, 2020.

[2] M. Goulet, L. Archambault, L. Beaulieu, and L. Gingras, "High resolution 2D dose measurement device based on a few long scintillating fibers and tomographic reconstruction," Medical Physics, vol. 39, no. 8, pp. 48404849, 2012.

[3] L. Desbat, S. Rit, R. Clackdoyle, P. Jalade, J. Ribouton, and P. Pittet, "Geometric tomography for measuring rectangular radiotherapy fields from six projections," in 2019 IEEE Nuclear Science Symposium and Medical Imaging Conference (NSS/MIC), 2019, pp. 1-4.

[4] R. J. Gardner and P. Gritzmann, Uniqueness and Complexity in Discrete Tomography. Boston, MA: Birkhäuser Boston, 1999, pp. 85-113.

[5] R. J. Gardner, Geometric tomography. Cambridge University Press Cambridge, 1995, vol. 6.

[6] R. Gardner and P. Gritzmann, "Discrete tomography: determination of finite sets by x-rays," Transactions of the American Mathematical Society, vol. 349, no. 6, pp. 2271-2295, 1997.

[7] S. Brunetti and A. Daurat, "Determination of Q-convex bodies by $\mathrm{x}$ rays," Electronic Notes in Discrete Mathematics, vol. 20, pp. 67 - 81, 2005, proceedings of the Workshop on Discrete Tomography and its Applications. 